\section{Subthreshold diode micropulse panretinal photocoagulation for proliferative diabetic retinopathy}

\begin{abstract}
Purpose To report the visual acuity and clinical outcomes of a pilot study of subthreshold diode micropulse (SDM) panretinal photocoagulation (PRP) for treatment of diabetic retinopathy.
\end{abstract}

Methods A retrospective chart review of all patients undergoing PRP for diabetic retinopathy between April 2000 and February 2003 was performed. Treated conditions ranged from severe non-proliferative to severe proliferative diabetic retinopathy. An SDM PRP protocol designed to avoid detectable laser lesions was employed. Treatment failure end points included the development of vitreous haemorrhage or the performance of vitrectomy.

Results Ninety-nine eyes of 63 patients undergoing SDM PRP were identified. Median follow-up was $\mathbf{1 . 0}$ year (range of 0.3-2.7 years). Treatment sessions per eye ranged from 1 to 6 (with a median of two sessions per eye). Overall visual acuity remained unchanged. The probability of treatment failure end points at 12 months posttreatment was $\mathbf{1 2 . 5 \%}$ for vitreous haemorrhage and $\mathbf{1 4 . 6 \%}$ for vitrectomy (from Kaplan-Meier survival analysis). Age, sex, diabetes type, and baseline retinopathy status were not significantly associated with the risk of either failure event. No treatment complications were observed. No eye demonstrated any laser lesion detectable clinically or by fluorescein angiography postoperatively.

Conclusion SDM pan retinal

photocoagulation minimized retinal damage and treatment complications in the management of high-risk non proliferative and proliferative diabetic retinopathy. Visual loss was prevented with a low rate of vitreous

JK Luttrull', DC Musch² and CA Spink ${ }^{1}$

haemorrhage and vitrectomy postoperatively. Further study of the safety, efficacy, and optimal treatment parameters of SDM pan retinal photocoagulation for diabetic retinopathy is warranted.

Eye (2008) 22, 607-612; doi:10.1038/sj.eye.6702725; published online 9 February 2007

\section{Introduction}

Shown conclusively in several landmark studies to reduce the risk of visual loss, visible end point (conventional) photocoagulation remains the primary treatment for diabetic retinopathy. ${ }^{1-6}$ However, the retinal destruction inherent in conventional photocoagulation may cause both short- and long-term visual loss and compromise other visual functions limiting the usefulness and effectiveness of treatment. ${ }^{7-17}$

Recently, two studies reported successful subthreshold treatment of diabetic macular oedema employing a micropulsed diode laser. $^{18,19}$ In one of these, treatment was performed without evidence of laser-induced retinal injury. ${ }^{19}$ Herein, we describe use of the same subthreshold diode micropulse (SDM) treatment strategy to perform panretinal photocoagulation (PRP) for severe nonproliferative and proliferative diabetic retinopathy.

\section{Methods}

Upon receiving authorization by the hospital Institutional Review Board, we reviewed the records of all patients treated for retinal vascular disease in a solo private vitreoretinal subspecialty practice in the period from April 2000 to February 2003. We identified 231
${ }^{1}$ Suite 230, Ventura, CA, USA

${ }^{2}$ Departments of Ophthalmology and Visual Sciences and Epidemiology, Schools of Medicine and Public Health, University of Michigan, Ann Arbor, MI, USA

Correspondence: JK Luttrull, 3160 Telegraph Road, Suite 230, Ventura, CA 93003,

USA

Tel: 8056500865 ;

Fax: 8056500865 .

E-mail: jkluttrull@

aol.com

Received: 22 June 2006 Accepted in revised form: 23 December 2006 Published online: 9 February 2007

Commercial interests: (1) None. (2) Consultant, IRIDEX, Mountain View, CA, USA. 
consecutive patients (348 eyes) treated with SDM for retinal vascular disease.

Eyes selected for this study had diabetic retinopathy ranging from severe non-proliferative to any degree of proliferative diabetic retinopathy (PDR) undergoing pan retinal SDM. Exclusion criteria included conventional PRP performed before April 2000 (56 eyes), less than 3 months follow-up after SDM PRP (15 eyes), and eyes with preoperative visual acuity of less than $5 / 200$ (25 eyes). Ninety-nine eyes from 63 patients were found to be eligible for this study.

Recorded information included age, race, sex, laterality, diabetes type, presence or absence of systemic hypertension, other medical conditions and use of medications, best corrected Snellen visual acuity, lens status, prior ocular surgery, presence or absence of anterior segment neovascularization, presence or absence of macular oedema, prior and/or subsequent macular laser treatment, presence or absence of vitreous haemorrhage, retinopathy severity, and performance of vitrectomy. SMD treatment parameters were noted for each eye.

Treatment failure end points selected for this study were either the development of postoperative vitreous haemorrhage or performance of vitrectomy. If vitreous haemorrhage developed, the occurrence and date of occurrence were noted. For patients with and without vitreous haemorrhage, data were recorded up to and including their most recent clinical examination. For patients requiring vitrectomy, the final follow-up visit recorded was the final examination before vitrectomy.

Diabetic retinopathy severity was determined from chart notes, fundus photographs and fundus drawings and categorized as follows: severe non-proliferative (SNDR) (severe generalized background retinopathy with additional findings of multiple retinal infarcts, and/or venous dilation, focal narrowing, or loops); low-risk PDR (LRPDR) (neovascularization less than Diabetic Retinopathy Study Research Group (DRS) definition of 'high risk' findings); high-risk PDR (HRPDR) (according to DRS threshold criterion); moderately severe PDR (MSPDR) ( 1-4 disc areas of disc and/or retinal neovascularization \pm vitreous

haemorrhage); and severe PDR (SPDR) ( $>4$ disc areas of neovascularization \pm vitreous haemorrhage) ${ }^{5}$

A positive treatment response for PDR was defined as the disappearance, regression or arrest and fibrosis of neovascularization postoperatively. For severe non-PDR a positive treatment response was inferred by lack of progression to proliferative retinopathy.

The time-related probability of treatment failure events - post-treatment vitreous haemorrhage and vitrectomy - was estimated using Kaplan-Meier survival analysis. Risk factors for these events were evaluated using Cox proportional hazards models. SAS 9.1 statistical software (Cary, NC, USA) was used for all data analysis.

\section{SDM treatment parameters}

All SDM PRP was performed in a single session by a single surgeon (JKL). Informed consent was obtained. Although conventional suprathreshold PRP was available to every patient, each patient was offered SDM PRP as less likely to require local anaesthesia, less likely to cause retinal damage or scarring, and potentially effective but possibly requiring additional/repeated treatments or abandonment in favour of conventional PRP if unsuccessful.

Topical anaesthesia was administered. An inverting wide-field contact lens (Volk 160 Panfundus lens, Volk Corporation, Mentor, OH, USA) was applied to the cornea with methylcellulose fluid. An $810 \mathrm{~nm}$ diode laser photocoagulator (Iris Medical OcuLight SLx, IRIDEX Corporation, Mountain View, CA, USA) was used in its MicroPulse operating mode. All patients were treated with a $500 \mu \mathrm{m}$ aerial spot size, $0.20 \mathrm{~s}$ pulse envelope duration, with an initial $2.0 \mathrm{~W}$ power setting. Within the laser pulse envelope, a 15\% duty cycle, each delivering a train of 100 sequential laser pulses of $300 \mu \mathrm{s}$ 'on' time separated by $1700 \mu \mathrm{s}$ 'off' time, was employed for each patient.

All areas of the fundus outside the posterior major vascular arcades as far as to the retinal periphery could be visualized were treated with a tight grid pattern of SDM. Owing to the absence of observable laser lesions at the time of treatment, the fundus was visually divided into sectors demarcated by the retinal vascular tree, facilitating creation of a short-term mental 'inventory' of the treated areas. These sectors were then treated in succession until PRP was complete. If the patient complained of discomfort, the power, spot size and/or pulse envelope duration were reduced until comfortable and treatment was completed. In contrast to typical visible end point argon laser PRP, the patient pain threshold with SMD PRP employing a $500 \mu \mathrm{m}$ aerial (1000 $\mu \mathrm{m}$ retinal) spot size was generally significantly lower than the threshold for creating visible retinal photocoagulation burns.

\section{Results}

Study patients ranged in age from 33 to 85 years, with a mean age of 60.5 years $(\mathrm{SD}=12.1$ years). Thirty-nine were male $(62 \%)$ and 24 female (38\%). Thirty-two patients were Caucasian (51\%), 23 Hispanic (37\%) and eight Asian (13\%). Twenty-six patients had type I diabetes mellitus (41\%) and 37 had type II (59\%) diabetes mellitus. Treated conditions ranged from severe 
non-proliferative to severe proliferative diabetic retinopathy (Table 1).

All patients elected SDMM PRP as an initial treatment. No patient required subsequent conventional PRP owing to failure to respond to SDM PRP. The number of SDM laser applications delivered at the initial treatment session ranged from 458 to 2001 (median 1425 per session), which was higher than the overall median number of applications per session across all sessions, 1218 (range 490-5093). The total number of laser applications delivered per eye ranged from 638 to 25466 with a median of 2234. The number of SDM PRP sessions per eye ranged from 1 to 6 , with a median of 2.0 sessions per eye. (Table 2) No patient required injection of local anaesthesia.

Follow-up after the initial SDM PRP session ranged from 98 to 997 days, with a mean of 390 days (1.1 years) $(\mathrm{SD}=239$ days $)$ and a median follow-up of 1 year. Eighteen of 99 eyes (18\%) presented with vitreous haemorrhage, and 81 eyes (81\%) without. Of the 81 eyes presenting without vitreous haemorrhage, 17 eyes (21\%) developed vitreous haemorrhage during follow-up. In eyes presenting without vitreous haemorrhage, the probability of vitreous haemorrhage at 1 year following initial SDM PRP for PDR was 12.5\% (Kaplan-Meier

Table 1 Proliferative DR change during follow-up (all eyes, $n=99)$ DR status during follow-up (best recorded)

\begin{tabular}{lrrrrrr}
\hline Initial DR status & $S N P D R$ & LRPDR & HRPDR & MSPDR & SPDR & Total \\
\hline SNPDR & 35 & 1 & 1 & 1 & 0 & 38 \\
LRPDR & 0 & 6 & 0 & 1 & 0 & 7 \\
HRPDR & 0 & 0 & 19 & 0 & 0 & 19 \\
MSPDR & 0 & 0 & 0 & 9 & 0 & 9 \\
SPDR & 0 & 0 & 0 & 0 & 26 & 26 \\
Total & 35 & 7 & 20 & 11 & 26 & 99
\end{tabular}

Abbreviations: DR, diabetic retinopathy; HRPDR, high risk PDR; LR, low risk proliferative DR (PDR); MSPDR, moderately severe PDR; SNPDR, severe non proliferative DR; SPDR, severe PDR. survival analysis) (Figure 1). Age, sex, diabetes type, and baseline diabetes status (PPDR vs PDR) were not significantly associated with risk of developing vitreous haemorrhage. PDR severity was a risk factor for vitreous haemorrhage of borderline significance, with a hazard ratio in the full model of $6.0(95 \% \mathrm{CI}, 0.7-48.6 ; P=0.09)$ indicating that eyes presenting with PDR were at sixfold risk of vitreous haemorrhage compared to eyes with SNDR.

Of the 35 eyes (35\%) presenting with or developing vitreous haemorrhage during follow-up, 10 (29 or $10 \%$ of all study eyes) underwent vitrectomy for non-clearing vitreous haemorrhage. This includes four of 18 eyes presenting with vitreous haemorrhage, and six of 17 eyes presenting without vitreous haemorrhage which developed vitreous haemorrhage following SDM PRP. An additional seven (7\%) study eyes underwent vitrectomy for reasons other than non-clearing vitreous haemorrhage. The length of time from initial SDM PRP to vitrectomy in all eyes ranged 134-313 days, mean 246 days. Overall, the probability of undergoing vitrectomy following SDM PRP for PDR was $14.6 \%$ at 12 months (Kaplan-Meier survival analysis). (Figure 2) Age, sex,

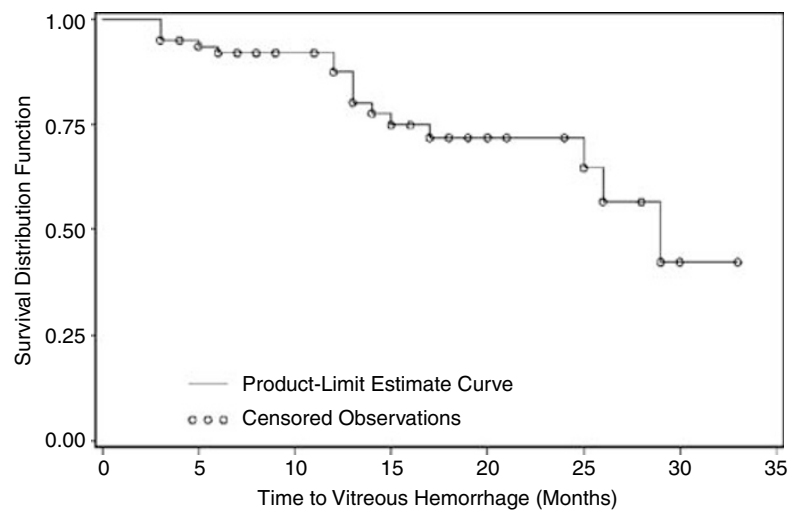

Figure 1 Kaplan-Meier survival analysis of time to vitreous haemorrhage following SDM PRP for PDR.

Table 2 Laser treatment parameters

\begin{tabular}{|c|c|c|c|}
\hline Parameter & Mean (SD) & Median & Range \\
\hline No. of laser sessions per eye & $2.2(1.3)$ & 2.0 & $1-6$ \\
\hline No. of laser applications delivered at the initial session & $1696(1914)$ & 1425 & 458-2001 \\
\hline Total no. of laser applications delivered per eye (all sessions) & $3003(3160)$ & 2234 & $638-25466$ \\
\hline Number of laser sessions per eye & Number & & Percentage \\
\hline One & 33 & & 33.3 \\
\hline Two & 35 & & 35.4 \\
\hline Three & 15 & & 15.2 \\
\hline Four & 9 & & 9.1 \\
\hline Five & 5 & & 5.0 \\
\hline Six & 2 & & 2.0 \\
\hline
\end{tabular}




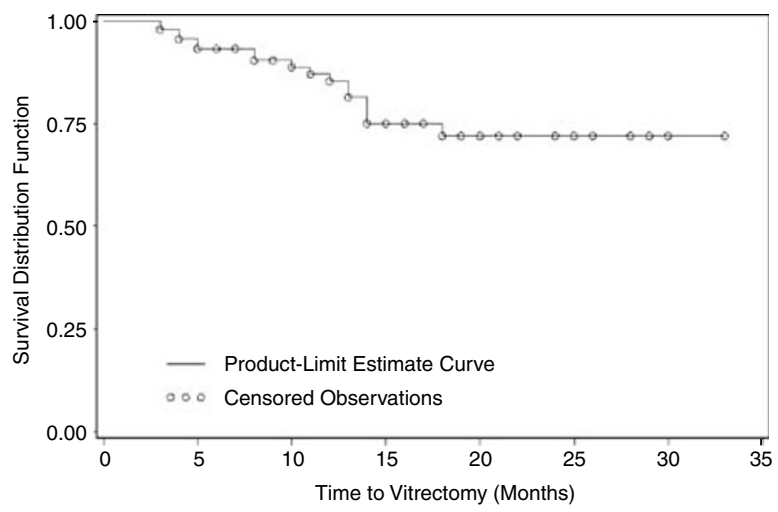

Figure 2 Kaplan-Meier survival analysis of time to vitrectomy following SDM PRP for PDR.

diabetes type, and diabetic retinopathy severity were not significantly associated with the risk of vitrectomy.

Four eyes presented with iris neovascularization (NVI). One additional eye developed NVI following initial treatment. In four of these five eyes, the iris neovascularization resolved or arrested. No studied eye required surgery for neovascular glaucoma. In three eyes (3\%), severity of retinopathy increased despite initial SDM PRP. Additional SDM PRP achieved arrest and regression of $\mathrm{NV}$ in these three eyes.

With respect to visual acuity changes of three or more Snellen lines, visual acuities on average remained unchanged during follow-up. Thirty-nine eyes (39\%) presented with visual acuities of 20/30 or better. During follow-up, 48 eyes ( $48 \%$ ) had best recorded visual acuities of $20 / 30$ or better. Of the 60 eyes presenting with visual acuities of $20 / 40$ or worse, eight eyes (13\%) showed three or more Snellen lines of visual improvement. Four eyes lost three or more Snellen lines of visual acuity by the last follow-up examination. Two eyes ended with visual acuity of less than 5/200, both due to macular ischemia. No eye suffered loss of light perception (Figure 3).

No complication of SDM PRP was observed. No eye demonstrated visible laser lesions or laser-induced chorioretinal scarring clinically or angiographically at any time postoperatively. No patient complained of postoperative pain or loss of visual acuity, accommodation, night vision, or visual field.

Postoperative inflammation was not observed in any eye. No eye demonstrated worsening of macular oedema following SDM PRP, and no eye without macular oedema developed macular oedema postoperatively.

\section{Discussion}

Despite the effectiveness of conventional photocoagulation, there is no evidence that the retinal

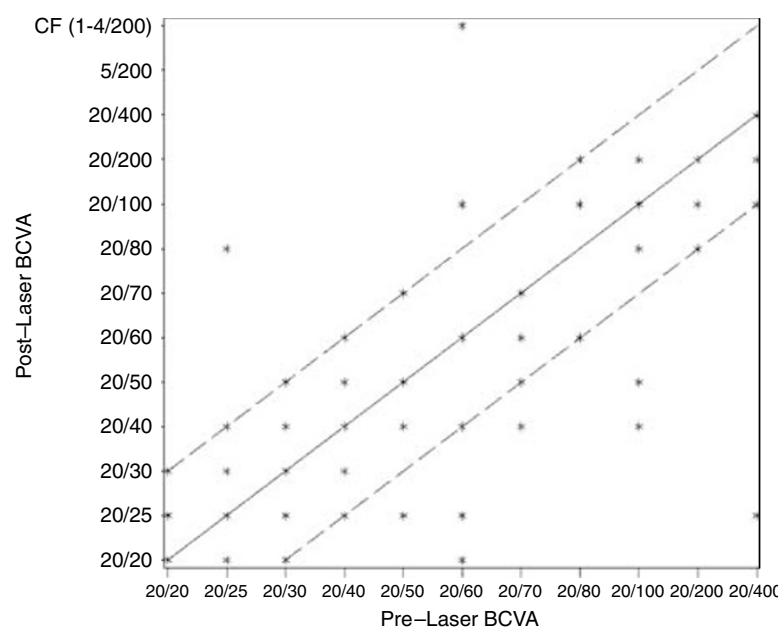

Figure 3 Scatter plot of initial visual acuity by best post-SDM PRP visual acuity scatter plot of initial vs post-SDM PRP visual acuity. Solid line defines agreement between pre- and post- SDM PRP visual acuity. Dotted lines define two Snellen line change in best-corrected visual acuity between pre- and post- SDM PRP. $\mathrm{SDM}$, subthreshold diode micropulse photocoagulation; PRP, PRP; BCVA, best corrected Snellen visual acuity; CF, finger counting.

destruction inherent in visible end point

photocoagulation is actually necessary to achieve a therapeutic benefit in the treatment of retinal vascular disease. ${ }^{5,7-17,19-22}$ With this in mind, we describe a novel atraumatic photocoagulation technique for treatment of diabetic retinopathy. We used a low-duty cycle micropulsed diode laser to perform subthreshold photocoagulation that does not cause retinal scarring. In so doing, we observed none of the complications associated with conventional visible end point photocoagulation. Our 'high-density/low-intensity' SDM PRP technique, employing a large retinal laser spot size and low laser irradiance level, appeared effective in the treatment of severe non-proliferative and proliferative diabetic retinopathy. ${ }^{19,23-27}$

Like previous studies of PRP for PDR, overall visual acuity was unchanged. ${ }^{28-30}$ However, the proportion of eyes with excellent visual acuity of 20/30 or better visual acuity increased from 39 to $48 \%$ during the course of the study, possibly due to avoidance of treatment complications that can reduce visual acuity postoperatively.

As the DRS and ETDRS the management of diabetic retinopathy has continued to evolve. In addition to recent advances in pharmacotherapy, vitrectomy techniques, and indications continue to improve and expand. ${ }^{31-36}$ Vitrectomy for diabetic retinopathy is now often undertaken earlier and for indications other than vitreous haemorrhage or traction macular detachment. Thus, it is difficult to directly compare this with past 
studies of conventional PRP for PDR. With this caveat in mind, we chose the occurrence of vitreous haemorrhage and the performance of vitrectomy as treatment 'failure' end points. Although the risk of vitreous haemorrhage increased with increasing severity of retinopathy, due to the limited number of eyes in each subgroup of DR severity we had insufficient power to evaluate differences in $\mathrm{VH}$ or vitrectomy between retinopathy categories. (Table 1) In this study, 17 of 81 eyes (21\%) presenting without vitreous haemorrhage developed vitreous haemorrhage. As the DRS and ETDRS, few reports have examined the efficacy of PRP for PDR in comparable patient populations. However, Kaiser et al, ${ }^{28}$ reported a similar incidence of de novo vitreous haemorrhage of $28 \%$ in 165 eyes followed 1 year after conventional argon laser PRP for PDR.

Vitrectomy was performed in $17 / 99$ (17\%) of study eyes. This includes $4 / 18(22 \%)$ of eyes presenting with vitreous haemorrhage and 6/17 (35\%) of eyes developing new vitreous haemorrhage. Thus $7 / 17$ (41\%) eyes in this study undergoing vitrectomy were operated for reasons other than non-clearing vitreous haemorrhage, most commonly premacular fibrosis. Although higher than the ETDRS vitrectomy incidence of $5.6 \%$, our $17 \%$ vitrectomy incidence ( $10 \%$ for non-clearing vitreous haemorrhage) is within the range reported in contemporary studies. ${ }^{28,37}$

Consistent with the observations of the DRS, ETDRS, and subsequent studies, the most common clinical response to SDM PRP was arrest or regression and fibrosis of neovascularization rather than disappearance. Compared to our experience with conventional PRP, the response to SDM PRP developed more gradually and without marked contraction of the neovascular tissue. SDM PRP may thus be especially useful in the management of eyes with extensive active neovascularization, which are more prone to retinal detachment following conventional PRP. ${ }^{16,38}$

Patient acceptance of SDM was high. SDM PRP was well tolerated in a single session with topical anaesthesia alone by all patients. One-third of eyes required a single treatment session, slightly less than the $45 \%$ reported for conventional PRP. ${ }^{20}$ However, re-treatments were well tolerated and could be performed as necessary due to the absence of retinal damage, scarring, inflammation, and associated complications. ${ }^{39}$ The $810 \mathrm{~nm}$ wavelength allowed treatment through dense nuclear sclerotic cataracts, preretinal fibrosis, vitreous haemorrhage, and thin preretinal blood.

Owing to lack of precedence and absence of a visible treatment end point, the SDM PRP treatment parameters we used in this study were arrived at empirically, designed to avoid creation of clinically detectable photocoagulation lesions. Subsequently, with Mainster and co-workers, ${ }^{19}$ we suggested rational bases for refinement of SDM treatment parameters, which may improve the effectiveness of SDM while still avoiding detectable retinal injury. We believe that the effectiveness of SDM PRP we have observed at the low laser irradiance levels employed in this and our previous report of SDM for diabetic macular oedema support the relevance of SDM theory in clinical practice. ${ }^{19,40,41}$

This study reviews the experience of a single surgeon employing a novel photocoagulation strategy in a clinical setting to treat diabetic retinopathy. Although visual acuity outcomes were assessed, other tests of retinal function such as visual field testing and electrophysiology were not performed. Owing to the absence of clinically notable retinal injury we suspect that such testing, which should be a part of any future study, may further differentiate the results of SDM PRP from conventional PRP. The present study is further limited by its small sample size, brief follow-up, and reliance upon historical controls resulting in difficulty comparing our findings to previous studies. However, the results suggest that with respect to preservation of visual acuity and the incidence of untoward postoperative events such as vitreous haemorrhage and performance of vitrectomy, SDM PRP may be useful in the treatment of severe non-proliferative and PDR while avoiding laser-induced retinal damage and the complications associated with conventional photocoagulation. The shift in the benefit/risk ratio afforded by the low treatment risk of SDM has altered our approach to the management of diabetic retinopathy. ${ }^{19,41}$ We believe that further study of SDM for treatment of diabetic retinopathy and other retinal vascular disorders in a randomized clinical trial is warranted.

\section{References}

1 Kahn HA, Hiller R. Blindness caused by diabetic retinopathy. Am J Ophthalmol 1974; 78: 58-67.

2 Kahn HA, Bradley RF. Prevalence of diabetic retinopathy. Age, sex, and duration of diabetes. Br J Ophthalmol 1975; 59: 345-349.

3 Aiello LM, Rand LI, Briones JC, Wafia MZ, Sebestyen JG. Diabetic retinopathy in Joslin clinic patients with adultonset diabetes. Ophthalmology 1981; 88: 619-623.

4 Photocoagulation for diabetic macular edema. Early Treatment Diabetic Retinopathy Study report number 1. Early Treatment Diabetic Retinopathy Study research group. Arch Ophthalmol 1985; 103: 1796-1806.

5 Diabetic Retinopathy Study Research Group. Photocoagulation treatment of proliferative diabetic retinopathy: the second report of Diabetic Retinopathy Study (DRS) findings. Ophthalmology 1978; 85: 82-106.

6 Early Treatment of Diabetic Retinopathy Study Research Group. Early photocoagulation for diabetic retinopathy. ETDRS Report Number 9. Ophthalmology 1991; 98(Suppl): 766-785. 
7 Schatz H, Madeira D, McDonald HR, Johnson RN Progressive enlargement of laser scars following grid laser photocoagulation for diffuse diabetic macular edema. Arch Ophthalmol 1991; 109: 1549-1551.

8 Morgan CM, Schatz H. Atrophic creep of the retinal pigment epithelium after focal macular photocoagulation. Ophthalmology 1989; 96: 96-103.

9 Early Treatment of Diabetic Retinopathy Study Research Group. Focal photocoagulation treatment of diabetic macular edema. Relationship of treatment effect to fluorescein angiographic and other retinal characteristics at baseline: ETDRS report no. 19. Early Treatment Diabetic Retinopathy Study Research Group. Arch Ophthalmol 1995; 113: 1144-1155.

10 Lewen RM. Subretinal neovascularization complicating laser photocoagulation of diabetic maculopathy. Ophthalmic Surg 1988; 19: 734-737.

11 Lewis H, Schachat AP, Haimann MH, Yeo JH, Quinlan P, Murphy RP et al. Choroidal neovascularization after laser photocoagulation for diabetic macular edema. Ophthalmology 1990; 97: 503-510; discussion 510-511.

12 Guyer DR, D'Amico DJ, Smith CW. Subretinal fibrosis after laser photocoagulation for diabetic macular edema. Am J Ophthalmol 1992; 113: 652-656.

13 Rutledge BK, Wallow IH, Poulsen GL. Sub-pigment epithelial membranes after photocoagulation for diabetic macular edema. Arch Ophthalmol 1993; 111: 608-613.

14 Higgins KE, Meyers SM, Jaffe MJ, Roy MS, de Monasterio FM. Temporary loss of foveal contrast sensitivity associated with panretinal photocoagulation. Arch Ophthalmol 1986; 104: 997-1003.

15 Henricsson M, Heiji A. The effect of panretinal photocoagulation on visual acuity, visual field and on subjective visual field impairment in preproliferative and early proliferative diabetic retinopathy. Acta Ophthalmol Otolaryngol 1977; 83: 476-487.

16 Diabetic Retinopathy Study Group. Photocoagulation treatment of proliferative diabetic retinopathy: relationship of adverse treatment effects to retinopathy severity. DRS Report Number 5. Dev Ophthalmol 1981; 2: 248-261.

17 Shimura M, Yasuda K, Nakazawa T, Tamai M. Visual dysfunction after panretinal photocoagulation in patients with severe diabetic retinopathy and good vision. Am J Ophthalmol 2005; 140: 8-15.

18 Laursen ML, Moeller F, Sander B, Sjoelie AK. Subthreshold micropulse diode laser treatment in diabetic macular oedema. Br J Ophthalmol 2004; 88: 1173-1179.

19 Luttrull JK, Musch DC, Mainster MA. Subthreshold diode micropulse photocoagulation for the treatment of clinically significant diabetic macular oedema. Br J Ophthalmol 2005; 89: 74-80.

20 Doft BH, Metz DJ, Kelsey SF. Augmentation laser for proliferative diabetic retinopathy that fails to respond to initial panretinal photocoagulation. Ophthalmology 1992; 99: 1728-1734.

21 Blankenship GW. Preoperative prognostic factors in diabetic pars plana vitrectomy. Ophthalmology 1982; 89: 1246-1249.

22 Ligget PE, Lean JS, Barlow WE, Ryan SJ. Intraoperative argon endophotocoagulation for recurrent vitreous hemorrhage after vitrectomy for diabetic retinopathy. Am J Ophthalmol 1987; 103: 146-149.

23 Pankratov MM. Pulsed delivery of laser energy in experimental thermal retinal photocoagulation. Proc Soc Photo-Optical Instrum Eng 1990; 1202: 205-213.
24 Sliney DH, Marshall J. Tissue specific damage to the retinal pigment epithelium: mechanisms and therapeutic implications. Lasers Light Ophthalmol 1992; 5: 17-28.

25 Roider J, Hillenkamp F, Flotte T, Birngruber R. Microphotocoagulation: selective effects of repetitive short laser pulses. Proc Natl Acad Sci USA 1993; 90: 8643-8647.

26 Roider J, Brinkmann R, Wirbelauer C, Laqua $\mathrm{H}$, Birngruber R. Retinal sparing by selective retinal pigment epithelial photocoagulation. Arch Ophthalmol 1999; 117: 1028-1034.

27 Mainster MA. Decreasing retinal photocoagulation damage: principles and techniques. Semin Ophthalmol 1999; 14: 200-209.

28 Kaiser RS, Maguire MG, Grunwald JE, Lieb D, Jani B, Brucker AJ et al. One-year outcomes of panretinal photocoagulation in proliferative diabetic retinopathy. Am J Ophthalmol 2000; 129: 1778-1785.

29 Dogru M, Nakamura M, Inoue M, Yamamoto M. Long-term visual outcome in proliferative diabetic retinopathy patients after panretinal photocoagulation. Jpn J Ophthalmol 1999; 43: 217-224.

30 Bandello R, Brancato R, Menchini U, Virgili G, Lanzetta P, Ferrari E. Light panretinal photocoagulation (LPRP) versus classic panretinal photocoagulation (CPRP) in proliferative diabetic retinopathy. Semin Ophthalmol 2001; 16: 12-18.

31 Jonas JB, Sofker A. Intraocular injection of crystalline cortisone as adjunctive treatment of diabetic macular edema. Am J Ophthalmol 2001; 132: 425-427.

32 Harbour JW, Smiddy WE, Flynn Jr HW, Rubsamen PE. Vitrectomy for diabetic macular edema associated with a thickened and taut posterior hyaloid membrane. Am J Ophthalmol 1996; 121: 405-413.

33 La Heij EC, Hendrikse F, Kessels AG, Derhaag PJ. Vitrectomy results in diabetic macular oedema without evident vitreomacular traction. Graefes Arch Clin Exp Ophthalmol 2001; 239: 264-270.

34 Otani T, Kishi S. A controlled study of vitrectomy for diabetic macular edema. Am J Ophthalmol 2002; 134: 214-219.

35 Diabetic Retinopathy Vitrectomy Study Research Group. Early vitrectomy for severe diabetic retinopathy in eyes with useful vision: results of a randomized clinical trial. DRVS Report number 3. Ophthalmology 1988; 95: 1307-1320.

36 Diabetic Retinopathy Vitrectomy Study Research Group. Early vitrectomy for severe vitreous hemorrhage in diabetic retinopathy: four year results of a randomized clinical trial. DRVS Report Number 5. Arch Ophthalmol 1990; 108: 958-964.

37 Early Treatment of Diabetic Retinopathy Study Research Group. Plars plana vitrectomy in the Early Treatment of Diabetic Retinopathy Study. ETDRS Report Number 17. Ophthamology 1992; 99: 1351-1357.

38 Doft BH, Blankenship G. Retinopathy risk factor regression after laser panretinal photocoagulation for proliferative diabetic retinopathy. Ophthalmology 1984; 91: 1453-1457.

39 McDonald HR, Schatz H. Macular edema following panretinal photocoagulation. Retina 1985; 98: 741-756.

40 Wilson AS, Hobbs BG, Shen WY, Sped TP, Schmidt U, Begley CG et al. Argon laser photocoagulation-induced modification of gene expression in the retina. Invest Ophthalmol Vis Sci 2003; 44: 1426-1434.

41 Luttrull JK, Spink CA. Serial optical coherence tomography of subthreshold diode micropulse photocoagulation for diabetic macular edema. Ophthalmic Surgery, Lasers and Imaging 2006; 37: 370-377. 